\title{
High expression of TXNDC11 indicated unfavorable prognosis of glioma
}

\author{
Peng Peng ${ }^{1}$, Fangling Cheng ${ }^{2}$, Yuting Dong ${ }^{3,4,5}$, Zirong Chen ${ }^{1}$, Xiaolin Zhang ${ }^{1}$, Dongsheng Guo ${ }^{1}$, \\ Xingjiang Yu' ${ }^{6}$, Yiyang $\mathrm{Lu}^{7}$, Yuyong $\mathrm{Ke}^{8}$, Bin Zhang ${ }^{3,5,9}$, Ximiao $\mathrm{He}^{3,4,5}$, Feng Wan ${ }^{1}$
}

${ }^{1}$ Department of Neurosurgery, Tongji Hospital of Tongji Medical College, Huazhong University of Science and Technology, Wuhan, China; ${ }^{2}$ Department of Surgery, Hepatic Surgery Center, Tongji Hospital of Tongji Medical College, Huazhong University of Science and Technology, Wuhan, China; ${ }^{3}$ Department of Physiology, School of Basic Medicine, Tongji Medical College, Huazhong University of Science and Technology, Wuhan, China; ${ }^{4}$ Center for Genomics and Proteomics Research, School of Basic Medicine, Tongji Medical College, Huazhong University of Science and Technology, Wuhan, China; ${ }^{5}$ Hubei Key Laboratory of Drug Target Research and Pharmacodynamic Evaluation, Huazhong University of Science and Technology, Wuhan, China; ${ }^{6}$ Department of Histology and Embryology, School of Basic Medicine, Tongji Medical College, Huazhong University of Science and Technology, Wuhan, China; ${ }^{7}$ School of Data Science, Chinese University of Hong Kong, Shenzhen, China; ${ }^{8}$ Department of Neurosurgery, Renmin Hospital of Yangxin County, Huangshi, China; ${ }^{9}$ The Institute for Brain Research, Collaborative Innovation Center for Brain Science, Huazhong University of Science and Technology, Wuhan, China

Contributions: (I) Conception and design: B Zhang, X He, F Wan; (II) Administrative support: Y Ke; (III) Provision of study materials or patients: P Peng, F Cheng, Y Dong; (IV) Collection and assembly of data: P Peng, F Cheng, Y Dong, Z Chen, X Zhang, D Guo; (V) Data analysis and interpretation: X Yu, Y Lu; (VI) Manuscript writing: All authors; (VII) Final approval of manuscript: All authors.

Correspondence to: Bin Zhang; Ximiao He. Department of Physiology, School of Basic Medicine, Tongji Medical College, Huazhong University of Science and Technology, Hangkong Road 13, Wuhan 430030, China. Email: binzhang@hust.edu.cn; XimiaoHe@hust.edu.cn; Feng Wan. Department of Neurosurgery, Tongji Hospital of Tongji Medical College, Huazhong University of Science and Technology, Jiefang Avenue 1095, Wuhan 430030, China. Email: wanruiyan@hotmail.com.

Background: Thioredoxin domain containing 11 (TXNDC11) has been implicated in numerous cancers. Nevertheless, the function of TXNDC11 in glioma is not well described. This study aimed to assess clinical significance of TXNDC11 in glioma based on bioinformatics analysis and immunohistochemical (IHC) staining.

Methods: GEPIA2, The Cancer Genome Atlas (TCGA), and Gene Expression Omnibus (GEO) databases were employed to detect the levels of TXNDC11 transcript in glioma. Gene expression profiles and data from the methylation chip with clinical details from TCGA and Chinese Glioma Genome Atlas (CGGA) of glioma samples were examined. The methylation of TXNDC11 in glioma was evaluated by 450K methylation chip data analysis. The pathways involved in TXNDC11 expression were screened by gene set enrichment analysis (GSEA). The correlation between TXNDC11 and immune cells was analyzed. Protein level of TXNDC11 was detected by IHC staining in glioma specimens.

Results: TXNDC11 was highly expressed in glioma, and high TXNDC11 expression was associated with poor overall survival (OS) and worse clinical prognostic variables. The methylation of cg04399632 was statistically different between glioma samples and normal samples, and was negatively correlated with TXNDC11 expression in glioma patients. Survival analysis demonstrated a poorer prognosis in glioma patients with cg04399632 hypomethylation. TXNDC11-high phenotype was associated with certain immunerelated pathways and other signaling pathways in glioma. The expression of TXNDC11 was correlated positively with M2 macrophage infiltration and negatively with M0 and M1 macrophage infiltration. IHC staining confirmed that TXNDC11 expression increased in higher-grade glioma.

Conclusions: High expression of TXNDC11 may predict unfavorable prognosis of glioma patients.

Keywords: Thioredoxin domain containing 11 (TXNDC11); prognosis; glioma; gene set enrichment analysis (GSEA); macrophage 
Submitted Jul 17, 2021. Accepted for publication Nov 12, 2021.

doi: $10.21037 /$ tcr-21-1326

View this article at: https://dx.doi.org/10.21037/tcr-21-1326

\section{Introduction}

Gliomas are the most prevalent primary brain tumors and one of the deadliest solid tumors with a high relapse rate and low chance of recovery (1). Despite the improvements in the treatment modalities in the past two decades, clinical therapeutic effect of glioma remains unsatisfactory. With the rapid advancement of biomedical approaches, many glioma biomarkers have been developed (2-5). However, efficient and accurate biomarkers that can predict the prognosis of glioma are few.

Thioredoxin domain containing 11 (TXNDC11), also recognized as EF-Hand Binding Protein 1, acts as a redox regulator involved in protein folding of thyroid oxidase (6). Recently, TXNDC11 has been described as a hub gene in gynecological cancer progression (7). In patients with endometrial carcinoma of the uterine corpus, higher TXNDC11 expression was correlated with prolonged overall survival (OS). Elevated expression of TXNDC11 also indicated a good prognosis in hepatocellular carcinoma patients (8). However, much less is known about the epigenetic and genetic status of TXNDC11 in gliomas.

The current study aimed to define the correlation between TXNDC11 expression and glioma progression, and identify potential prognostic value of TXNDC11 based on The Cancer Genome Atlas (TCGA), the Gene Expression Omnibus (GEO), and the Chinese Glioma Genome Atlas (CGGA). We found that TXNDC11 was highly expressed in glioma and associated with reduced OS and higher WHO grades. Immunostaining confirmed that higher grade was associated with higher expression of TXNDC11 in samples collected at our hospital. Further, gene set enrichment analysis (GSEA) indicated that high expression of TXNDC11 was correlated with immune-related pathways and other signaling pathways such as M2 phenotype macrophage infiltration.

We present the following article in accordance with the REMARK reporting checklist (available at http://dx.doi. org/10.21037/tcr-21-1326).

\section{Methods}

\section{Dataset generation}

Microarray data on glioma patients were retrieved from the public GEO database (https://www.ncbi.nlm.nih.gov/ geo) (9) with access number GSE68848 (10). The dataset GSE68848 was used for TXNDC11 expression analysis between glioma tissues compared with non-tumor tissues. In addition, data on the gene expression profile comprising clinical data from glioblastoma (GBM) (HTSeq-FPKM) and low-grade glioma (LGG) projects were collected from the TCGA (https://cancergenome.nih.gov/) database (11). Moreover, the dataset of mRNAseq_325 of 325 glioma samples with mRNA data and clinical data was acquired from the CGGA database (http://www.cgga.org.cn/) (12). The TCGA and CGGA data were further examined for the correlations between the expression of TXNDC11 and clinical features (age, WHO grade, histology, etc.). The methylation chip data was downloaded from TCGA to analyze the methylation of TXNDC11 in gliomas.

\section{Immunobistochemical (IHC) staining}

The study was conducted in accordance with the Declaration of Helsinki (as revised in 2013). The study was approved by ethics board of Tongji Hospital, Tongji Medical College, Huazhong University of Science and Technology and informed consent was taken from all the patients. Seventyeight specimens of gliomas were collected at Tongji Hospital, Tongii Medical College, Huazhong University of Science and Technology from September 2014 to September 2016. Tissue sections $(4 \mu \mathrm{m}$ thick) were stained with primary rabbit antiTXNDC11 antibody (Sigma-Aldrich, St. Louis, MO, USA; Cat\# HPA041174) overnight at $4{ }^{\circ} \mathrm{C}$ and then incubated with biotinylated goat anti-Rabbit secondary antibodies for 1 hour. Finally, the sections were detected with SignalStain ${ }^{\circledR}$ DAB (Cell Signaling Technology, Danvers, MA, USA) and counterstained with QS haematoxylin (Vector Laboratories, 
Burlingame, CA, USA).

\section{GSEA}

GSEA (4.0.3) was performed to examine biological pathways with statistically relevant differences between high and low TXNDC11 expression groups. The gene-set permutations were executed 1,000 times for each sample. Gene sets have been shown to be significantly improved with a typical $\mathrm{P}$ value $<0.05$ and a false discovery rate $(\mathrm{FDR})<0.05$.

\section{Immune response gauging of TIICs via CIBERSORTT in glioma}

CIBERSORT (13) (https://cibersort.stanford.edu/) is an algorithm for deconvolution based on gene expression, and has been applied to analyze cell heterogeneity $(14,15)$. To analyze tumor-infiltrating immune cells (TIIC), TCGA gene expression datasets were configured and uploaded to the CIBERSORT web portal with standard signature matrix. The correlation with TXNDC11 was then calculated.

\section{Statistical analysis}

Statistical analyses were performed using $\mathrm{R}$ software v3.6.2. Mann-Whitney $U$ and logistic regression tests were used to evaluate the associations between TXNDC11 and clinicopathologic characteristics. Cox regression analyses and Kaplan-Meier method were used to analyze the effect of TXNDC11 on OS and other clinical variables. The correlation coefficient analysis was used to assess the correlation between TXNDC11 expression and immune cell types. $\mathrm{P}$ value $<0.05$ was regarded to be statistically significant.

\section{Results}

\section{TXNDC11 transcript levels in glioma based on databases}

TXNDC11 transcript levels in gliomas (LGGs and GBMs) and matched normal samples were analyzed with the online tool GEPIA2. The boxplots indicated that TXNDC11 expression was upregulated in LGGs and GBMs compared to the matched normal samples (Figure 1A,1B).

TXNDC11 expression data were collected from TCGA for 698 glioma samples. In tumor tissues, TXNDC11 was significantly overexpressed compared to normal tissues
(Figure $1 C ; \mathrm{P}<0.001)$. Furthermore, for validation we used GSE68848 dataset from the GEO database (Figure 1D; $\mathrm{P}<0.05)$. These results showed increased TXNDC11 transcript levels in glioma.

\section{Characteristics of TCGA and CGGA glioma patients}

We selected 1,114 gliomas of all grades from TCGA as a training cohort and 325 gliomas of all grades from CGGA for validation (Table 1). The median age (years) of patients was 51 (from 10 to 89) in TCGA and 42 (from 8 to 79) in CGGA. Males accounted for $58.4 \%$ in TCGA and $62.5 \%$ in CGGA. There were 249 grade II, 265 WHO grade III and 596 grade IV gliomas in TCGA (GBMs). CGGA dataset included 103 grade II, 79 grade III, and 139 grade IV gliomas. For isocitrate dehydrogenases (IDH) mutation, $373(33.48 \%)$ tumors were identified in TCGA and 517 (46.41\%) cases lack IDH details. In CGGA, all the 325 samples were detected for IDH mutation with 175 (53.85\%) tumors mutated. For 1p19q-codeletion status, noncodeletion accounted for $44.43 \%$ in TCGA and $76.92 \%$ in CGGA.

\section{Association of TXNDC11 expression and clinicopathologic features of glioma}

We compared the expression of TXNDC11 in gliomas of different grades (Figure 2), and found that high expression of TXNDC11 was significantly correlated with age $(\mathrm{P}<0.05)$, histologic type $(\mathrm{P}<0.05)$ and $\mathrm{WHO}$ grade $(\mathrm{P}<0.05)$ (Figure $2 A, 2 C, 2 F)$, while not associated with gender in the TGGA dataset (Figure 2B). In TCGA, the expression of TXNDC11 in IDH1 mutated samples was significantly lower than that of the wildtype $(\mathrm{P}<0.05)$, and the expression of TXNDC11 in $1 \mathrm{p} 19 \mathrm{q}$ non-codeletion samples was significantly higher than that of the codeletion samples $(\mathrm{P}<0.05)$ (Figure 2D,2E). These results were validated in the CGGA (Figure $3 A-3 F$ ).

Univariate logistic regression analysis indicated that TXNDC11 expression was correlated with poor clinical pathological variables (Table 2). High expression of TXNDC11 in glioma was significantly correlated with age [ $\geq 51$ vs. $<51, \mathrm{OR}=1.89,95 \% \mathrm{CI}$ : $(1.38-2.58), \mathrm{P}<0.001$ ], gender [male vs. female, OR $=0.69,95 \%$ CI: $(0.51-0.94)$, $\mathrm{P}<0.05]$, WHO grade [III vs. II, OR $=1.52,95 \%$ CI: $(1.07-$ 2.16), $\mathrm{P}<0.05$; IV vs. II, OR =3.00, 95\% CI: (1.99-4.57), $\mathrm{P}<0.001]$, histology type [GBM $v s$. astrocytoma, $\mathrm{OR}=1.59$, 95\% CI: (1.03-2.47), $\mathrm{P}<0.05$; GBM vs. oligoastrocytoma, $\mathrm{OR}=2.36,95 \%$ CI: $(1.47-3.81), \mathrm{P}<0.001$; and GBM $v$. 

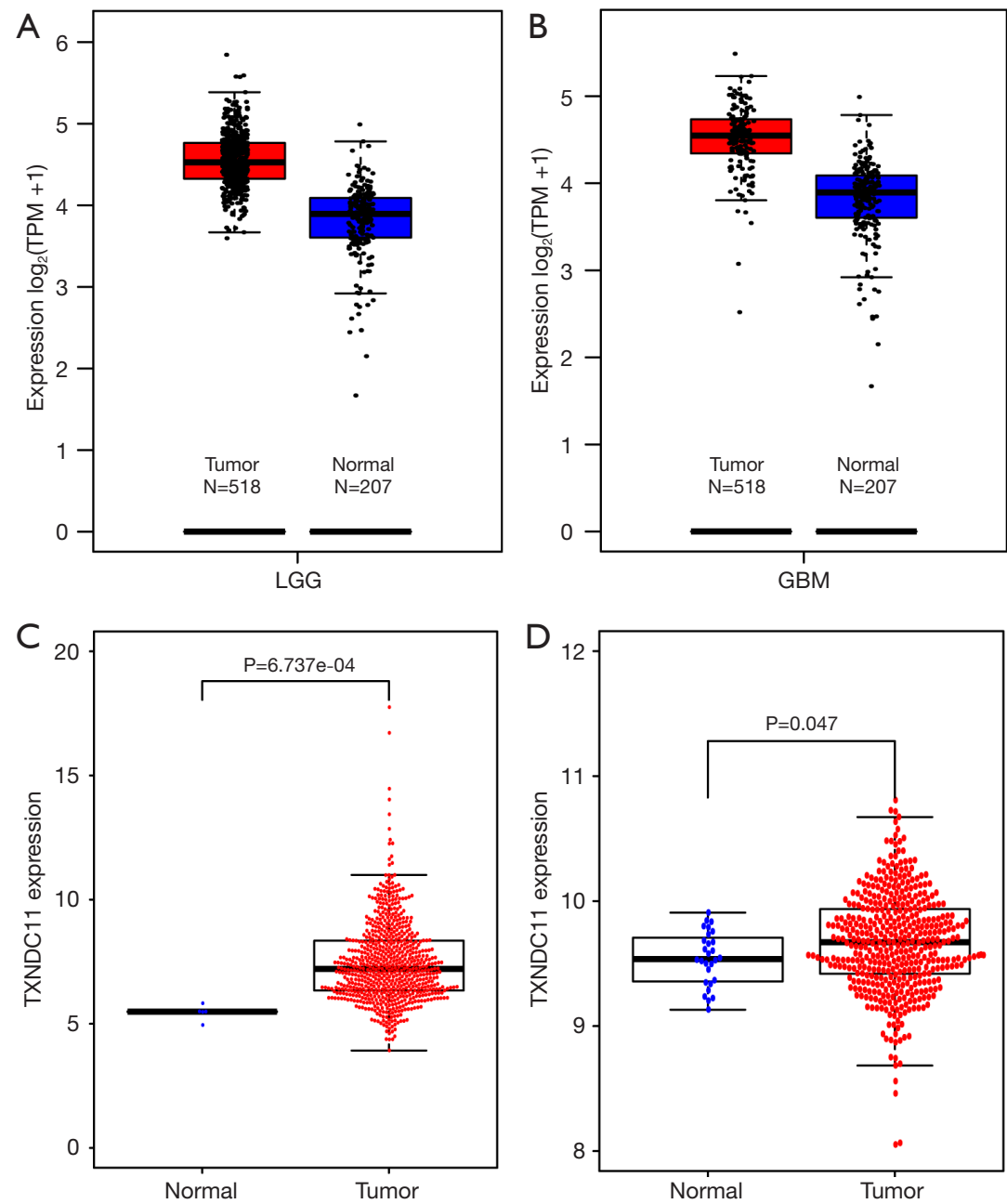

Figure 1 TXNDC11 expression was upregulated in glioma. (A,B) TXNDC11 expression was upregulated in LGGs and GBMs compared with the matched normal samples (analyzed with the online tool GEPIA2). (C,D) TCGA cohort, and GSE68848 dataset from GEO confirmed that TXNDC11 expression was upregulated in glioma. TXNDC11, thioredoxin domain containing 11; LGGs, low-grade gliomas; GBMs, glioblastomas; TCGA, The Cancer Genome Atlas; GEO, Gene Expression Omnibus.

oligodendroglioma, OR =3.44, $95 \%$ CI: $(2.22-5.38)$, $\mathrm{P}<0.001$ ], IDH1 mutation [wildtype vs. mutant, $\mathrm{OR}=2.14$, 95\% CI: (1.53-3.0), $\mathrm{P}<0.001], 1 \mathrm{p} 19 \mathrm{q}$-codeletion-status [non-codeletion $v s$. codeletion, $\mathrm{OR}=3.8,95 \% \mathrm{CI}$ : $(2.6-$ 5.63), $\mathrm{P}<0.001]$.

\section{Association of TXNDC11 expression and survival outcomes of glioma}

We examined OS and TXNDC11 expression in CGGA and TCGA databases to explore the predictive consequences of TXNDC11 in glioma prognosis. After excluding patients with missing OS data, Kaplan-Meier analysis showed that the prognosis of glioma patients with high TXNDC11 expression was poorer than that of patients with low TXNDC11 expression (Figure 4A,4B; $\mathrm{P}<0.001$ ).

Univariate Cox regression showed that high TXNDC11 expression was significantly associated with weaker OS [HR $=1.14,95 \%$ CI: $(1.07-1.22), \mathrm{P}<0001]$ (Table 3). Other clinicopathologic parameters associated with poor survival were age $[\mathrm{HR}=1.07,95 \% \mathrm{CI}:(1.06-1.08)]$, WHO grade [HR $=4.69,95 \%$ CI: (3.85-5.71)], and histological type [HR $=1.95$, 95\% CI: $(1.70-2.23)$ ] (all with $\mathrm{P}<0.001)$. Multivariate Cox analysis showed that TXNDC11 was independently correlated with OS, with HR of 1.09 [95\% CI: (1.00-1.18), $\mathrm{P}=0.04]$, along with age and $\mathrm{WHO}$ grade. 
Table 1 Baseline patient characteristics

\begin{tabular}{|c|c|c|}
\hline $\begin{array}{l}\text { Clinical } \\
\text { characteristics }\end{array}$ & TCGA $(n=1,114), n(\%)$ & CGGA $(n=325), n(\%)$ \\
\hline \multicolumn{3}{|l|}{ Age } \\
\hline Median [range] & $51[10-89]$ & 42 [8-79] \\
\hline \multicolumn{3}{|l|}{ Gender } \\
\hline Male & $651(58.4)$ & $203(62.5)$ \\
\hline Female & 460 (41.3) & $122(37.5)$ \\
\hline Missing & $3(0.27)$ & 0 \\
\hline \multicolumn{3}{|l|}{ WHO grade } \\
\hline II & $249(22.4)$ & $103(31.7)$ \\
\hline III & 265 (23.8) & $79(24.3)$ \\
\hline IV & $596(53.5)$ & $139(42.8)$ \\
\hline Missing & $4(3.6)$ & $4(1.2)$ \\
\hline \multicolumn{3}{|l|}{ Histology } \\
\hline$A$ & $194(17.4)$ & $118(36.31)$ \\
\hline $\mathrm{O}$ & $191(17.2)$ & $64(19.69)$ \\
\hline OA & $130(11.7)$ & NA \\
\hline GBM & $596(53.5)$ & $139(42.77)$ \\
\hline Missing & $3(0.27)$ & $4(1.23)$ \\
\hline \multicolumn{3}{|l|}{ IDH1 mutation } \\
\hline Mutation & $373(33.48)$ & $175(53.85)$ \\
\hline Wildtype & $224(20.11)$ & $149(45.85)$ \\
\hline Missing & $517(46.41)$ & $1(0.3)$ \\
\hline \multicolumn{3}{|c|}{ 1p19q-codeletion-status } \\
\hline Codeletion & $169(15.17)$ & $67(20.62)$ \\
\hline Non-codeletion & $495(44.43)$ & $250(76.92)$ \\
\hline Missing & $450(40.4)$ & $8(2.46)$ \\
\hline
\end{tabular}

TCGA, The Cancer Genome Atlas; CGGA, Chinese Glioma Genome Atlas; IDH, isocitrate dehydrogenases.

\section{The correlation between TXNDC11 methylation and prognosis of glioma}

The $450 \mathrm{~K}$ methylation chip data analysis revealed that the methylation of cg04399632 (the CpG site located in the S shore region of $\mathrm{CpG}$ island of TXNDC11) was statistically different between glioma and normal samples (Figure $5 A$ ). In GBM and LGG patients, the methylation of this site was negatively correlated with TXNDC11 expression
(Figure 5B-5D). According to the methylation at this site, GBM and LGG patients were divided into hypomethylation group and hypermethylation group. Survival analysis showed that hypermethylation glioma patients had a favorable prognostic value than hypomethylation patients $(\mathrm{P}<0.05)$ (Figure $5 E, 5 F)$.

\section{TXNDC11 related signaling patbways based on GSEA}

The unfavorable prognosis of glioma patients with high TXNDC11 expression may be related to signaling pathways commonly involved in cancer initiation and progression. GSEA was used to screen signaling pathways between low and high TXNDC11 expression samples. In TXNDC11 high expression cohorts, numerous signaling pathways, particularly inflammation, and immunity-related pathways, were greatly enhanced, including antigen processing and presentation, leukocyte transendothelial migration, B-cell receptor, cytokine-cytokine receptor interaction, Fc gamma $\mathrm{R}$-mediated phagocytosis, cell-mediated cytotoxicity of natural killer (NK), T-cell receptor and pancreatic receptor (Figure 6A, Table 4).

Correlation analysis showed that TXNDC11 transcription levels in TCGA were positively correlated with infiltration of M2 macrophages, monocytes, CD8 T cells, NK cells, but were negatively correlated with infiltration of M0 macrophages and M1 macrophages (Figure 6B).

\section{Protein expression of TXNDC11 in glioma specimens and HPA database}

Finally, we collected clinical samples and performed IHC staining. The analysis of TXNDC11 expression in 78 glioma specimens of grade II $(n=22)$, grade III $(n=13)$, and grade IV $(\mathrm{n}=43)$ showed increased TXNDC11 expression in high grade tumors (Figure $7 A, 7 B$ ). Similar findings were reported by examining IHC data from the HPA database (https://www. proteinatlas.org/), with $66.7 \%$ of LGG and $87.5 \%$ of highgrade glioma (HGG) being positive for TXNDC11 staining.

\section{Discussion}

Due to therapeutic resistance and high recurrence rate of the infiltrative gliomas after concurrent radio- and chemo- therapies, individualized treatment based on molecular targets has gained more attention. In recent years, important prognosis related molecular aberrations of gliomas have been found, including $1 \mathrm{p} / 19 \mathrm{q}$ codeletion, IDH mutation, MGMT promotor methylation, TERT 

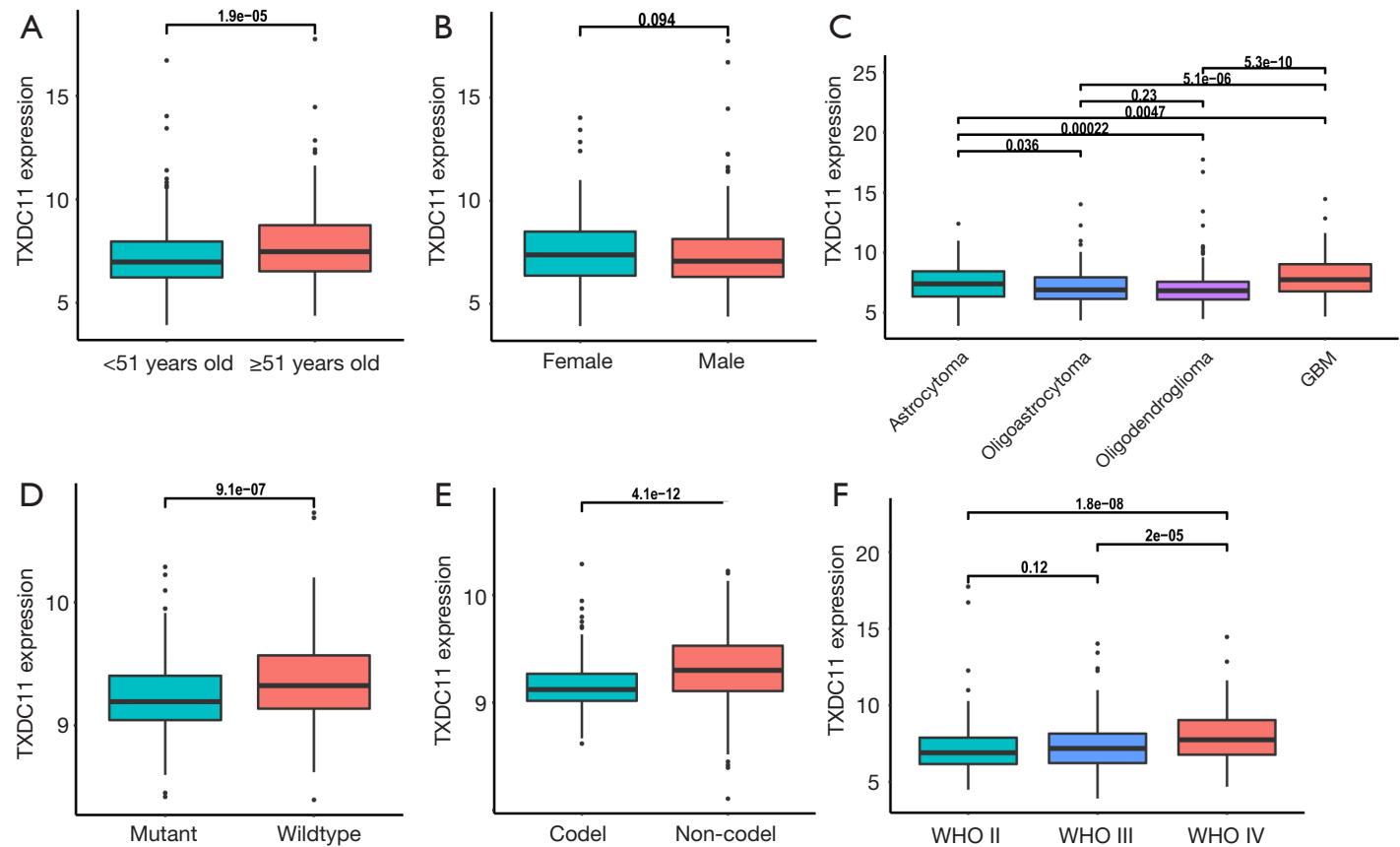

Figure 2 Associations between TXNDC11 expression and clinicopathologic variables in TCGA cohort. (A) Age, (B) gender, (C) histological type, (D) IDH1 mutation status, (E) 1p19q-codeletion-status, (F) WHO grade. TXNDC11, thioredoxin domain containing 11; TCGA, The Cancer Genome Atlas; IDH, isocitrate dehydrogenases.
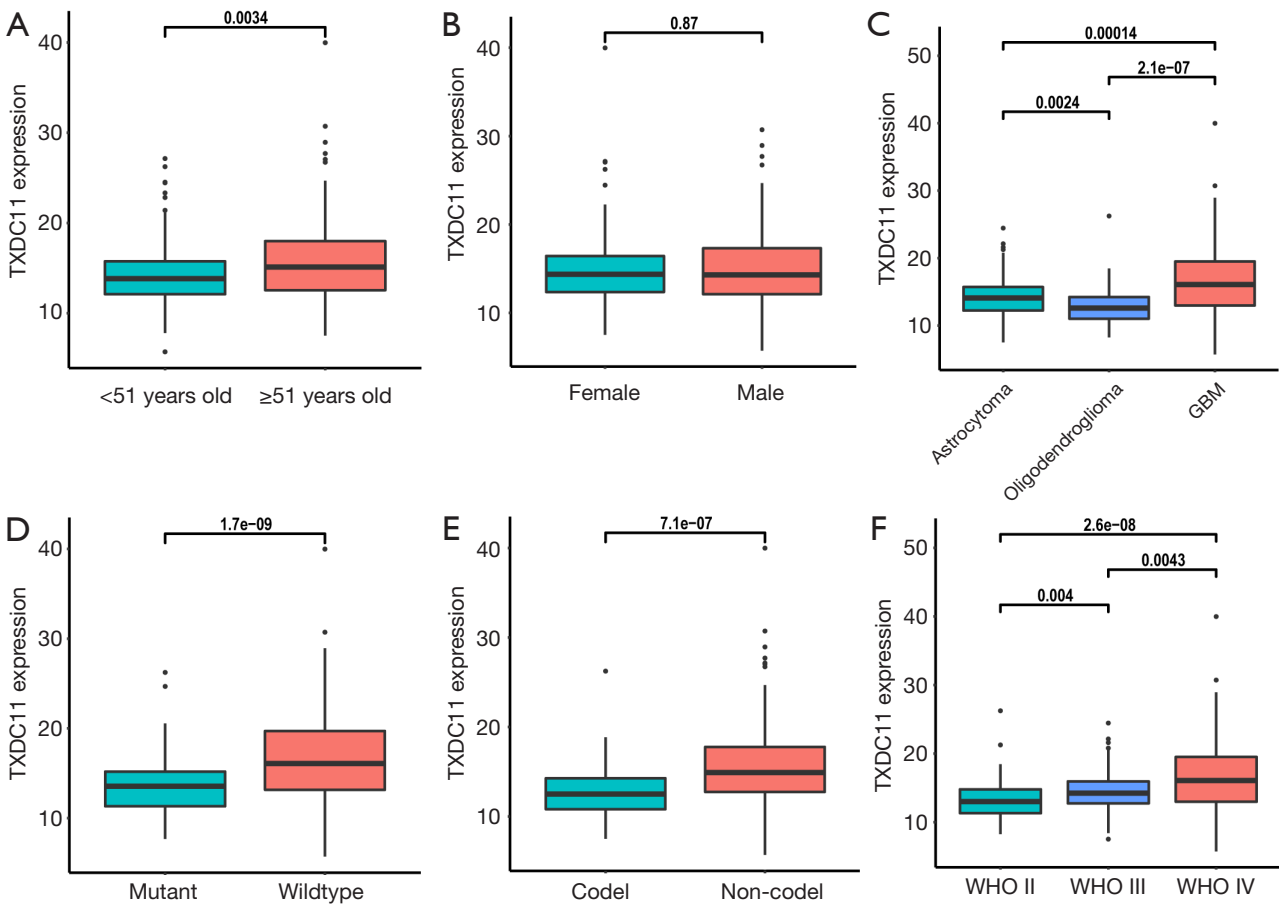

Figure 3 Associations between TXNDC11 expression and clinicopathologic variables in CGGA-325 cohort. (A) Age, (B) gender, (C) histological type, (D) IDH1 mutation status, (E) 1p19q-codeletion-status, (F) WHO grade. TXNDC11, thioredoxin domain containing 11; CGGA, Chinese Glioma Genome Atlas; IDH, isocitrate dehydrogenases. 
Table 2 Association of TXNDC11 expression in TCGA with clinical-pathological characteristics

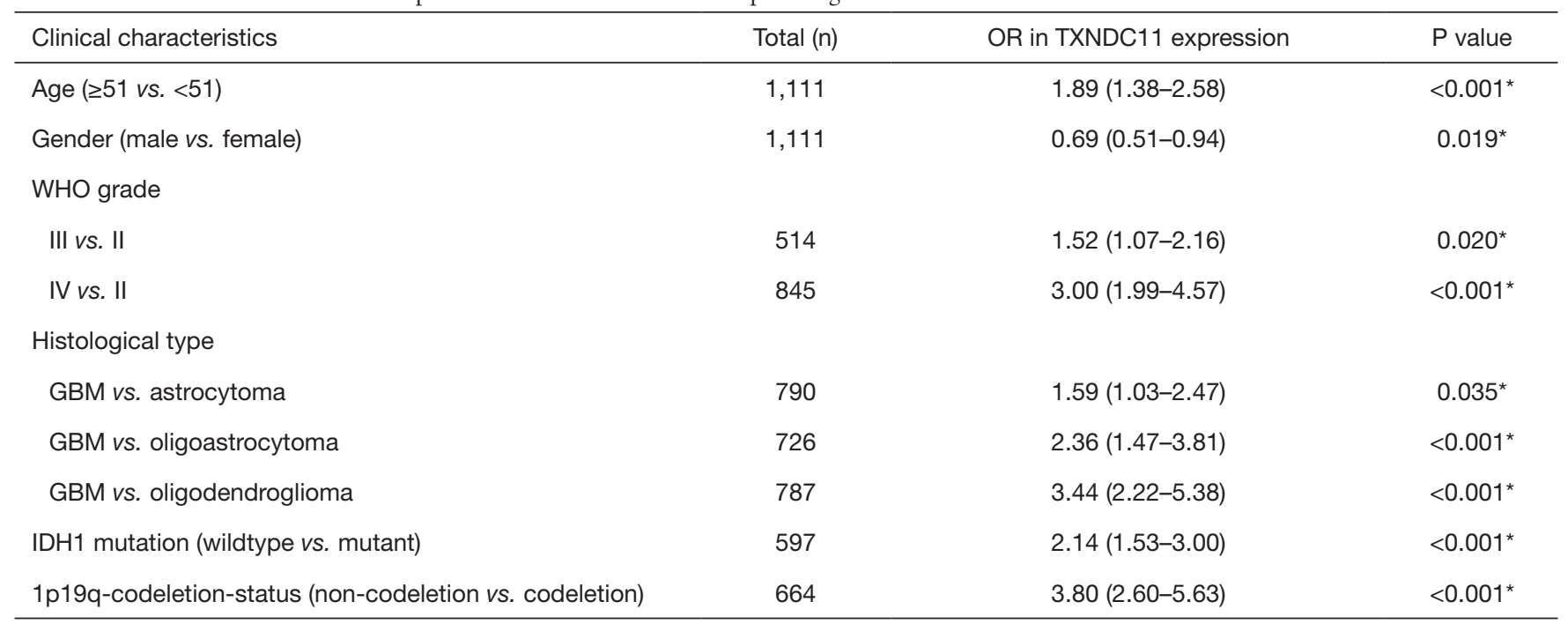

*, P<0.05. TXNDC11, thioredoxin domain containing 11; TCGA, The Cancer Genome Atlas; GBM, glioblastoma; IDH, isocitrate dehydrogenases.


Figure 4 Association of TXNDC11 expression and OS of glioma patients. (A) TCGA cohort. (B) CGGA cohort. TXNDC11, thioredoxin domain containing 11; OS, overall survival; TCGA, The Cancer Genome Atlas; CGGA, Chinese Glioma Genome Atlas.

Table 3 Univariate and multivariate analysis of clinicopathologic characteristics and OS in TCGA cohort

\begin{tabular}{|c|c|c|c|c|}
\hline Characteristics & \multicolumn{2}{|c|}{ Univariate analysis } & \multicolumn{2}{|c|}{ Multivariate analysis } \\
\hline Age & $<0.001^{*}$ & $1.07(1.06-1.08)$ & $<0.001^{*}$ & $1.04(1.03-1.05)$ \\
\hline Gender & 0.1095 & $1.23(0.95-1.59)$ & 0.56 & $1.08(0.84-1.40)$ \\
\hline WHO grade & $<0.001^{*}$ & $4.69(3.85-5.71)$ & $<0.001^{*}$ & $3.75(2.87-4.91)$ \\
\hline TXNDC11 expression & $<0.001^{*}$ & $1.14(1.07-1.22)$ & $0.04^{*}$ & $1.09(1.00-1.18)$ \\
\hline
\end{tabular}

*, P<0.05. OS, overall survival; TCGA, The Cancer Genome Atlas; TXNDC11, thioredoxin domain containing 11. 
A


B

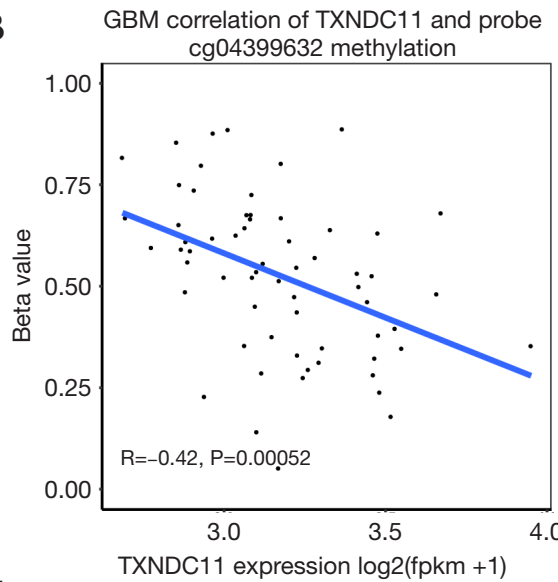

E



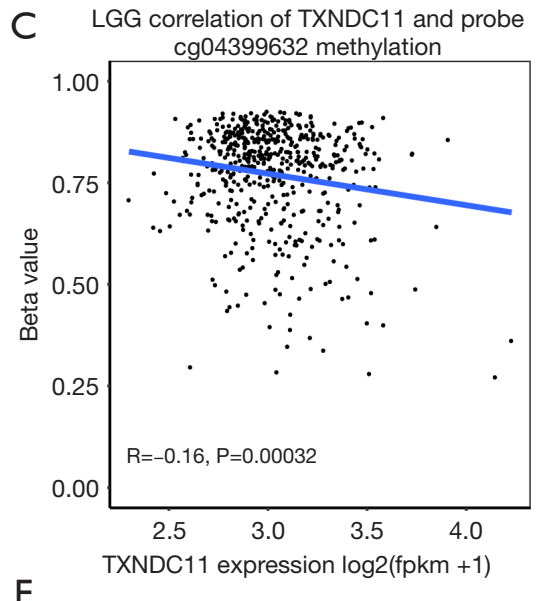

$\mathrm{F}$

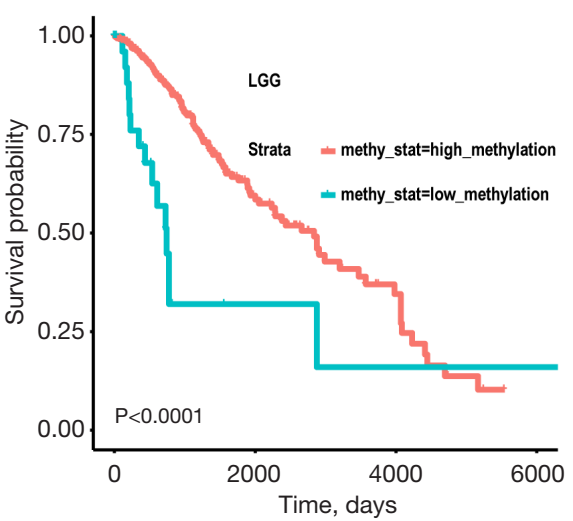

Figure 5 The correlation between TXNDC11 methylation and OS of glioma patients. (A) The methylation of cg04399632 in glioma and normal tissues. The correlation between TXNDC11 expression and the methylation of cg04399632 in GBM (B), LGG (C) patients, and all gliomas (D). Influence of the methylation on OS of glioma patients in GBM (E) and LGG (F) patients. TXNDC11, thioredoxin domain containing 11; OS, overall survival; LGG, low-grade glioma; GBM, glioblastoma.

mutation, EGFR amplification or EGFR VIII mutation (2).

In this study, we reported that TXNDC11 had higher expression in glioma than normal tissues and was correlated with clinical features such as older ages, higher WHO grade, $1 \mathrm{p} 19 \mathrm{q}$ non-codeletion and IDH wildtype. Survival analyses and Cox regression analyses confirmed that patients with higher TXNDC11 expression had a shorter OS, and TXNDC11 was an independent prognostic indicator of OS. Therefore, TXNDC11 might be a potential oncogene in glioma.

DNA methylation is a crucial epigenetic regulation mechanism for gene expression in cancers (16). The methylation status of MGMT promoter in gliomas can predict patient survival and response to the treatment by temozolomide (17-19). In this study, we found that gliomas had a lower methylation level at cg04399632 and the methylation level of this site was negatively correlated with
TXNDC11 expression. The methylation level at cg04399632 not only affected TXNDC11 mRNA expression, but also was positively correlated with patient survival.

Accumulating evidence has shown that tumor development and infiltration were related to oxidative stress (20-22). The excessive generation of cellular reactive oxygen species (ROS) and antioxidant system dysfunction cause oxidative stress (23). Free radicals and ROS serve as critical signaling molecules in immune process and inflammation $(24,25)$. TXNDC11 is a redox regulator that regulates redox status in the plasma membrane, cytosol, endoplasmic reticulum, and the nucleus $(6,26,27)$. Fu et al. showed that secretion of TXNRD1 was elevated in hepatocellular carcinoma patients under oxidative stress and inflammation (28). We assumed that TXNDC11 may contribute to glioma development via the immune process and inflammation mechanism. We used TCGA data for GSEA, 


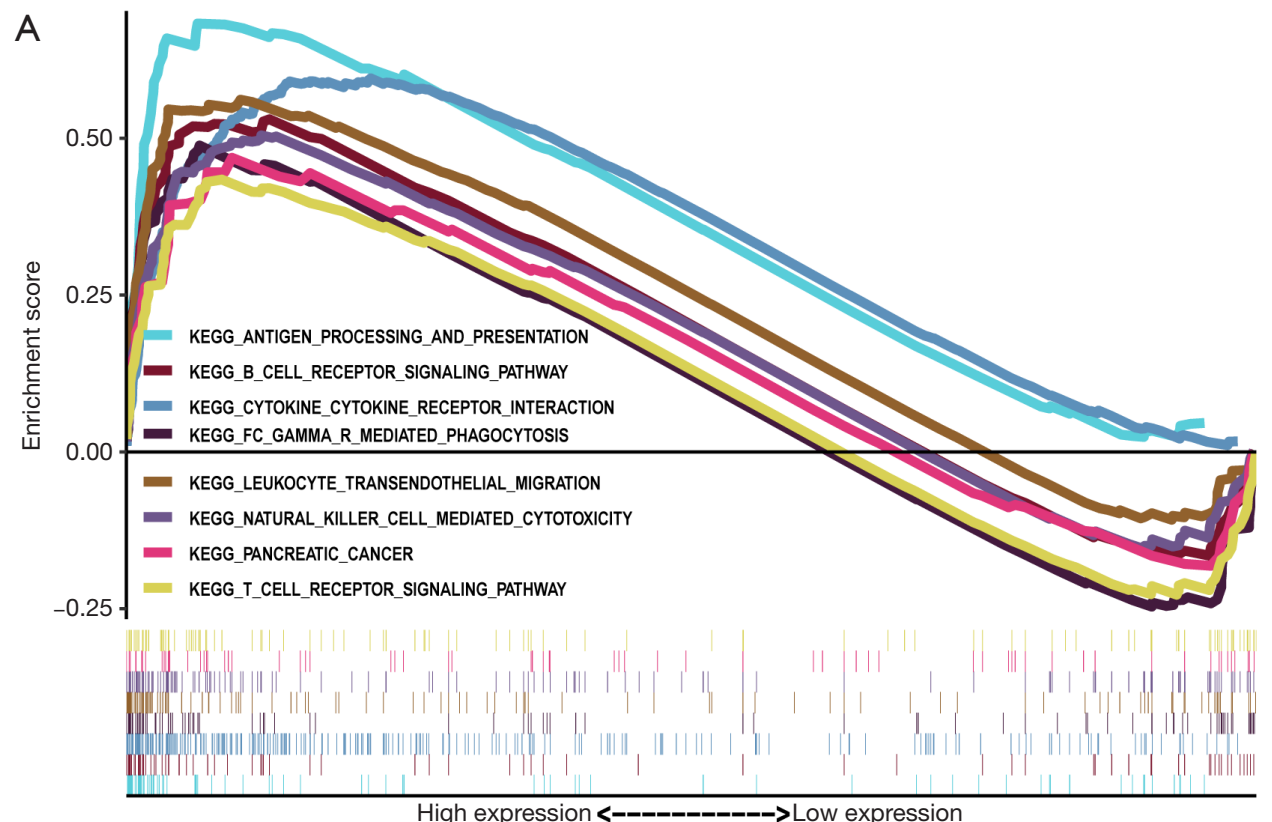

High expression <--_-------> Low expression
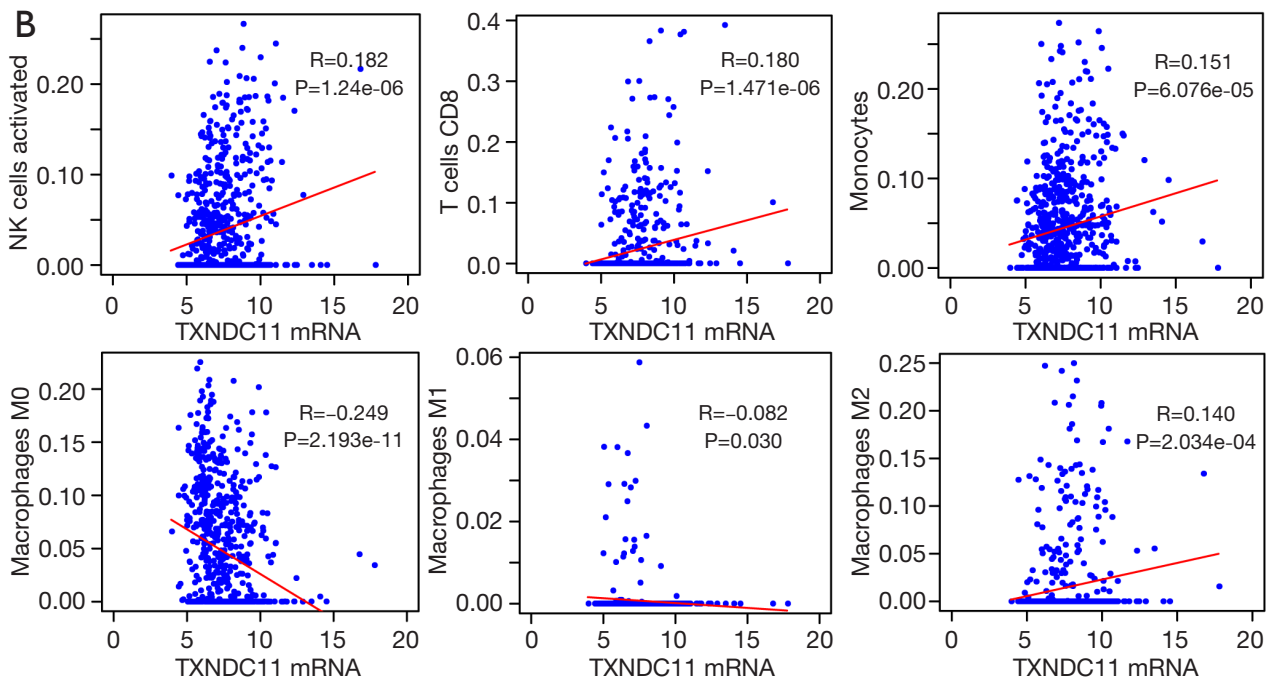

Figure 6 Correlations of TXNDC11 expression with immune cells in glioma. (A) GSEA analysis of signaling pathways enriched in glioma samples with high expression of TXNDC11. (B) TXNDC11 mRNA levels in TCGA were positively correlated with infiltration of M2 macrophages, monocytes, CD8 T cells, NK cells, but were negatively correlated with infiltration of M0 macrophages and M1 macrophages. TXNDC11, thioredoxin domain containing 11; GSEA, gene set enrichment analysis; TCGA, The Cancer Genome Atlas; NK, natural killer.

and found that inflammation and immune-related pathways, along with other pathways commonly involved in cancers, were associated with TXNDC11 expression.

Previous histopathological and flow cytometry studies revealed that various immune cells such as granulocytes, resident central nervous system (CNS) (microglia) and peripheral macrophages [glioma-associated macrophages (GAMs)], T lymphocytes, and myeloid-derived suppressor cells (MDSCs) were involved in tumor microenvironment (29-32). Heimberger et al. found a positive association between the intra-tumoral number of $\mathrm{CD}^{+}$cells and the survival of patients with glioma (33). Consistent with their report, we found the correlation between TXNDC11 transcript level and immune cell infiltration, such as CD8 T cells, NK cells activated, monocytes and macrophages.

GAMs constitute a significant immune cell population 
Table 4 Gene sets enriched in high TXNDC11 expression phenotype

\begin{tabular}{|c|c|c|c|c|}
\hline MSigDB collection & Gene set name & NES & NOM $P$ value & FDR q value \\
\hline \multirow{6}{*}{ Kegg.v6.2.symbols.gmt } & KEGG_CYTOKINE_CYTOKINE_RECEPTOR_INTERACTION & 1.986 & 0.002 & 0.007 \\
\hline & KEGG_B_CELL_RECEPTOR_SIGNALING_PATHWAY & 1.835 & 0.006 & 0.018 \\
\hline & KEGG_T_CELL_RECEPTOR_SIGNALING_PATHWAY & 1.568 & 0.042 & 0.081 \\
\hline & KEGG_FC_GAMMA_R_MEDIATED_PHAGOCYTOSIS & 1.754 & 0.018 & 0.028 \\
\hline & KEGG_LEUKOCYTE_TRANSENDOTHELIAL_MIGRATION & 1.976 & 0.002 & 0.007 \\
\hline & KEGG_PANCREATIC_CANCER & 1.728 & 0.012 & 0.033 \\
\hline
\end{tabular}

Gene sets with NOM $\mathrm{P}$ value $<0.05$ and FDR q value $<0.05$ were considered as significantly enriched. TXNDC11, thioredoxin domain containing 11; NES, normalized enrichment score; NOM, nominal; FDR, false discovery rate.



B

\begin{tabular}{|c|c|}
\hline WHO grade & TXNDC11 positive (\%) \\
\hline WHO II & $12 / 22(54.5)$ \\
\hline WHO II & $8 / 13(61.5)$ \\
\hline WHO IV & $36 / 43(83.7)$ \\
\hline
\end{tabular}
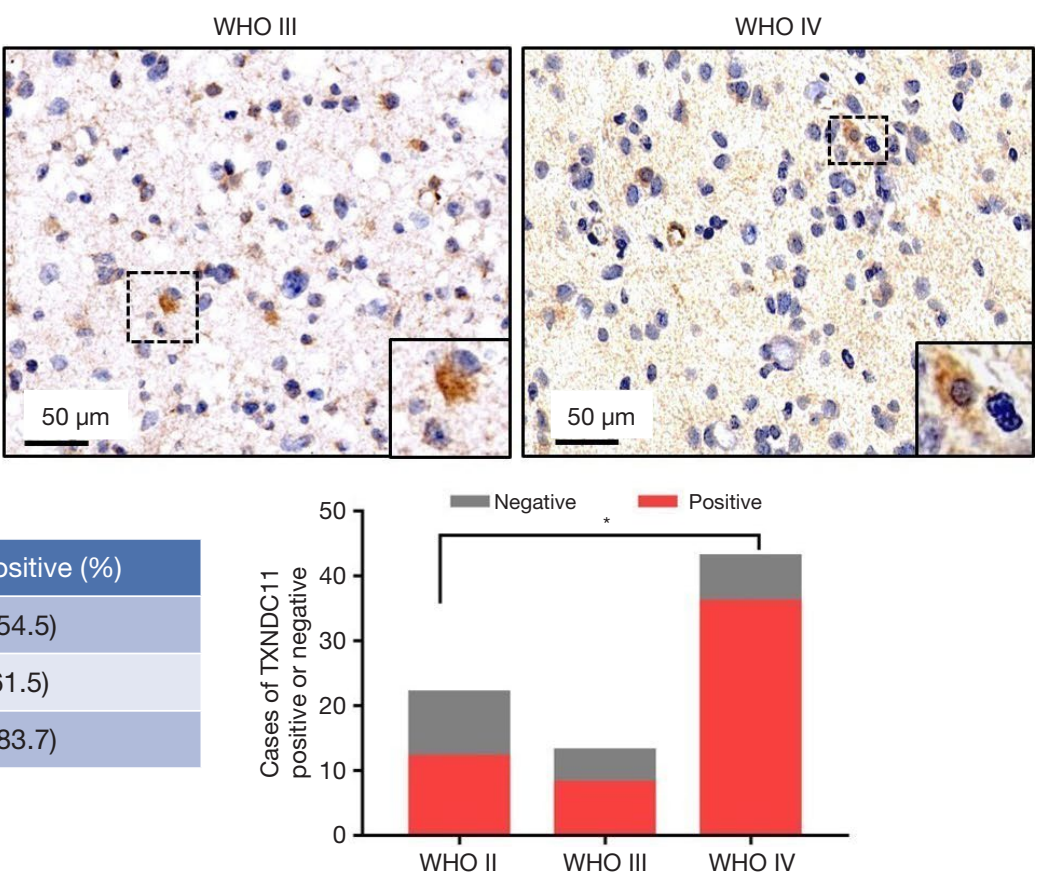

Figure 7 TXNDC11 expression increased in higher-grade glioma. (A) IHC analysis of TXNDC11 in glioma. Representative images were shown. Two times magnification of the box in the lower right corner. (B) Tumor type and TXNDC11 expression in glioma. The fractions and percentages of TXNDC11-positive tumors were shown. *, $\mathrm{P}<0.05$. TXNDC11, thioredoxin domain containing 11; IHC, immunohistochemical.

in gliomas and contribute to tumor growth and neovascularization (21,34-36). Lu-Emerson et al. found that increased GAM number was correlated with poor survival of GBM patients after anti-angiogenic therapy, which suggested that GAMs might participate in the escape of tumor cells from anti-angiogenic therapy, and therefore represent a potential biomarker of therapy resistance and a therapeutic target for GBMs (37). Moreover, M0 macrophages were negatively related to the prognosis of glioma patients, and M2-GAMs could promote the stemness and migration of glioma cells $(38,39)$. In this study, M0 and M1 macrophages were negatively correlated with TXNDC11 transcript level, while M2 macrophages were positively correlated with TXNDC11 transcript 
level. Therefore, the role of TXNDC11 in dictating GAM phenotypes and glioma progression need further studies.

In summary, we showed that TXNDC11 expression was upregulated in glioma and may contribute to unfavorable outcomes. Glioma patients with hypermethylation of TXNDC11 at cg04399632 site had a better prognosis than those with hypomethylation. TXNDC11 might be an important regulator of immune microenvironment of gliomas. Taken together, elevated expression of TXNDC11 may predict unfavorable prognosis of glioma patients.

\section{Acknowledgments}

We thank Elsevier China for professional editing service. Funding: This study was supported by grant of the Natural Science Foundation of China (NSFC: 82072795).

\section{Footnote}

Reporting Checklist: The authors have completed the REMARK reporting checklist. Available at https://dx.doi. org/10.21037/tcr-21-1326

Data Sharing Statement: Available at https://dx.doi. org/10.21037/tcr-21-1326

Conflicts of Interest: All authors have completed the ICMJE uniform disclosure form (available at https://dx.doi. org/10.21037/tcr-21-1326). The authors have no other conflicts of interest to declare.

Ethical Statement: The authors are accountable for all aspects of the work in ensuring that questions related to the accuracy or integrity of any part of the work are appropriately investigated and resolved. The study was conducted in accordance with the Declaration of Helsinki (as revised in 2013). The study was approved by ethics board of Tongji Hospital, Tongji Medical College, Huazhong University of Science and Technology and informed consent was taken from all the patients.

Open Access Statement: This is an Open Access article distributed in accordance with the Creative Commons Attribution-NonCommercial-NoDerivs 4.0 International License (CC BY-NC-ND 4.0), which permits the noncommercial replication and distribution of the article with the strict proviso that no changes or edits are made and the original work is properly cited (including links to both the formal publication through the relevant DOI and the license). See: https://creativecommons.org/licenses/by-nc-nd/4.0/.

\section{References}

1. Wen PY, Reardon DA. Neuro-oncology in 2015: Progress in glioma diagnosis, classification and treatment. Nat Rev Neurol 2016;12:69-70.

2. Siegal T. Clinical Relevance of Prognostic and Predictive Molecular Markers in Gliomas. Adv Tech Stand Neurosurg 2016;(43):91-108.

3. Wang Z, Zhang C, Liu X, et al. Molecular and clinical characterization of PD-L1 expression at transcriptional level via 976 samples of brain glioma. Oncoimmunology 2016;5:e1196310.

4. Ozlem OZ. A Novel Mutation in Neurofibromatosis Type 1 with Optic Glioma. Oncologie 2020;22:155-60.

5. Li G, Wang Z, Zhang C, et al. Molecular and clinical characterization of TIM-3 in glioma through 1,024 samples. Oncoimmunology 2017;6:e1328339.

6. Wang D, De Deken X, Milenkovic M, et al. Identification of a novel partner of duox: EFP1, a thioredoxin-related protein. J Biol Chem 2005;280:3096-103.

7. Zhang $X$, Wang Y. Identification of hub genes and key pathways associated with the progression of gynecological cancer. Oncol Lett 2019;18:6516-24.

8. Cho SY, Kim S, Son MJ, et al. Clinical Significance of the Thioredoxin System and Thioredoxin-DomainContaining Protein Family in Hepatocellular Carcinoma. Dig Dis Sci 2019;64:123-36.

9. Edgar R, Domrachev M, Lash AE. Gene Expression Omnibus: NCBI gene expression and hybridization array data repository. Nucleic Acids Res 2002;30:207-10.

10. Madhavan S, Zenklusen JC, Kotliarov Y, et al. Rembrandt: helping personalized medicine become a reality through integrative translational research. Mol Cancer Res 2009;7:157-67.

11. Gao C, Zhuang J, Zhou C, et al. Prognostic value of aberrantly expressed methylation gene profiles in lung squamous cell carcinoma: A study based on The Cancer Genome Atlas. J Cell Physiol 2019;234:6519-28.

12. Zhao Z, Meng F, Wang W, et al. Comprehensive RNAseq transcriptomic profiling in the malignant progression of gliomas. Sci Data 2017;4:170024.

13. Gentles AJ, Newman AM, Liu CL, et al. The prognostic landscape of genes and infiltrating immune cells across human cancers. Nat Med 2015;21:938-45.

14. Ali HR, Chlon L, Pharoah PD, et al. Patterns of 
Immune Infiltration in Breast Cancer and Their Clinical Implications: A Gene-Expression-Based Retrospective Study. PLoS Med 2016;13:e1002194.

15. Bense RD, Sotiriou C, Piccart-Gebhart MJ, et al. Relevance of Tumor-Infiltrating Immune Cell Composition and Functionality for Disease Outcome in Breast Cancer. J Natl Cancer Inst 2016;109:djw192.

16. Phillips T. The role of methylation in gene expression. Nature Education 2008;1:116.

17. Hegi ME, Diserens AC, Gorlia T, et al. MGMT gene silencing and benefit from temozolomide in glioblastoma. N Engl J Med 2005;352:997-1003.

18. Stupp R, Mason WP, van den Bent MJ, et al. Radiotherapy plus concomitant and adjuvant temozolomide for glioblastoma. N Engl J Med 2005;352:987-96.

19. Stupp R, Hegi ME, Mason WP, et al. Effects of radiotherapy with concomitant and adjuvant temozolomide versus radiotherapy alone on survival in glioblastoma in a randomised phase III study: 5-year analysis of the EORTC-NCIC trial. Lancet Oncol 2009;10:459-66.

20. Wu L, Wang F, Xu J, et al. PTPN2 induced by inflammatory response and oxidative stress contributed to glioma progression. J Cell Biochem 2019;120:19044-51.

21. Goyal S, Chatterjee S, Gupta N. Analysis of Antioxidant Potential of Trigonella foenum-graecum (L.) Extract Against Tumorigenesis. Oncologie 2021;23:89-104.

22. Park HK, Hong JH, Oh YT, et al. Interplay between TRAP1 and Sirtuin-3 Modulates Mitochondrial Respiration and Oxidative Stress to Maintain Stemness of Glioma Stem Cells. Cancer Res 2019;79:1369-82.

23. Liang J, Cao R, Wang X, et al. Mitochondrial PKM2 regulates oxidative stress-induced apoptosis by stabilizing Bcl2. Cell Res 2017;27:329-51.

24. Qiao C, Yang J, Shen Q, et al. Traceable Nanoparticles with Dual Targeting and ROS Response for RNAi-Based Immunochemotherapy of Intracranial Glioblastoma Treatment. Adv Mater 2018;30:e1705054.

25. Coutinho de Souza P, Smith N, Atolagbe O, et al. OKN007 decreases free radical levels in a preclinical F98 rat glioma model. Free Radic Biol Med 2015;87:157-68.

26. Hanschmann EM, Godoy JR, Berndt C, et al. Thioredoxins, glutaredoxins, and peroxiredoxins--molecular mechanisms and health significance: from cofactors to antioxidants to redox signaling. Antioxid Redox Signal 2013;19:1539-605.

27. Timms RT, Menzies SA, Tchasovnikarova IA, et al. Genetic dissection of mammalian ERAD through comparative haploid and CRISPR forward genetic screens. Nat Commun 2016;7:11786.
28. Fu B, Meng W, Zeng X, et al. TXNRD1 Is an Unfavorable Prognostic Factor for Patients with Hepatocellular Carcinoma. Biomed Res Int 2017;2017:4698167.

29. Fecci PE, Mitchell DA, Whitesides JF, et al. Increased regulatory $\mathrm{T}$-cell fraction amidst a diminished CD4 compartment explains cellular immune defects in patients with malignant glioma. Cancer Res 2006;66:3294-302.

30. Lohr J, Ratliff T, Huppertz A, et al. Effector T-cell infiltration positively impacts survival of glioblastoma patients and is impaired by tumor-derived TGF- $\beta$. Clin Cancer Res 2011;17:4296-308.

31. Alexiou GA, Vartholomatos G, Karamoutsios A, et al. Circulating progenitor cells: a comparison of patients with glioblastoma or meningioma. Acta Neurol Belg 2013;113:7-11.

32. Wainwright DA, Dey M, Chang A, et al. Targeting Tregs in Malignant Brain Cancer: Overcoming IDO. Front Immunol 2013;4:116.

33. Heimberger AB, Abou-Ghazal M, Reina-Ortiz C, et al. Incidence and prognostic impact of FoxP3 + regulatory $\mathrm{T}$ cells in human gliomas. Clin Cancer Res 2008;14:5166-72.

34. Esiri MM, Morris CS. Immunocytochemical study of macrophages and microglial cells and extracellular matrix components in human CNS disease. 2. Non-neoplastic diseases. J Neurol Sci 1991;101:59-72.

35. Geranmayeh F, Scheithauer BW, Spitzer C, et al. Microglia in gemistocytic astrocytomas. Neurosurgery 2007;60:159-66; discussion 166.

36. Gieryng A, Pszczolkowska D, Walentynowicz KA, et al. Immune microenvironment of gliomas. Lab Invest 2017;97:498-518.

37. Lu-Emerson C, Snuderl M, Kirkpatrick ND, et al. Increase in tumor-associated macrophages after antiangiogenic therapy is associated with poor survival among patients with recurrent glioblastoma. Neuro Oncol 2013;15:1079-87.

38. Zhong QY, Fan EX, Feng GY, et al. A gene expressionbased study on immune cell subtypes and glioma prognosis. BMC Cancer 2019;19:1116.

39. Liu Z, Kuang W, Zhou Q, et al. TGF- $\beta 1$ secreted by M2 phenotype macrophages enhances the stemness and migration of glioma cells via the SMAD2/3 signalling pathway. Int J Mol Med 2018;42:3395-403.

Cite this article as: Peng $\mathrm{P}$, Cheng F, Dong $\mathrm{Y}$, Chen Z, Zhang X, Guo D, Yu X, Lu Y, Ke Y, Zhang B, He X, Wan F. High expression of TXNDC11 indicated unfavorable prognosis of glioma. Transl Cancer Res 2021;10(12):5040-5051. doi: $10.21037 /$ tcr-21-1326 